\title{
Fisura labiopalatina bilateral y su abordaje odontológico multidisciplinario. Reporte de caso
}

\section{Bilateral cleft lip and palate and its multidisciplinary dental approach. Case report}

\begin{abstract}
Resumen
La fisura labiopalatina es u na malformación c ongénita c raneofacial que se desarrolla a partir de defectos embriológicos durante la formación de la cara. Las anomalías se presentan fundamentalmente en el labio superior, la premaxila, el paladar y en el piso nasal. A continuación se presenta un caso clínico de una paciente que ingresó a los 7 años de edad diagnosticada con fisura de labio y paladar bilateral tratada en la Unidad de Malformaciones Craneofaciales de la Facultad de Odontología de la Universidad de Chile. La paciente presentaba una relación clase III esqueletal, falta de desarrollo del tercio medio facial, posición protruida de la premaxila, una maloclusión molar Clase III bilateral acompañada de malposición y apiñamiento dentario. Se realizó tratamiento ortopédico de expansión maxilar y posteriormente ortodóncico con aparatología fija. Posterior a la extracción de los dientes supernumerarios, cirugía de injerto óseo y gingivoperiosteoplastía, se derivó a un rehabilitador quien realizó un puente adhesivo y carillas dentales, devolviendo la estética al sector anterosuperior. Para finalizar, se instaló en la paciente placas removibles de contención tanto superior como inferior. De esta manera, se obtuvieron resultados satisfactorios en términos de una oclusión funcional, estable y estética.
\end{abstract}

Palabras clave: Fisura del paladar; Ortodoncia; Rehabilitación bucal.

\section{Caso Clínico}

Noemí Leiva Villagra ${ }^{1, a, b}$, Loreto Saavedra Layera $^{1, a, c}$, Francisca Carranza Morales ${ }^{1, a}$

${ }^{1}$ Facultad de Odontología. Universidad de Chile. Santiago, Chile.

a Cirujana Dentista

${ }^{\mathrm{b}}$ Especialista de Ortodoncia y Ortopedia Maxilofacial

c Diplomada en Ortodoncia Preventiva e Interceptiva

\section{Correspondencia:}

Noemí Leiva Villagra

Correo electrónico: leivanoemi@yahoo.com

General Holley 2381, oficina 606, Providencia, Santiago, Chile.

\section{Coautores: \\ Loreto Saavedra Layera \\ loreslayera@gmail.com \\ Francisca Carranza Morales \\ facarranza@miuandes.cl}

Conflicto de intereses: Los autores declaran no tener conflictos de interés.

Fuente de financiamiento: Autofinanciado.

Fecha de recepción: 27/08/17

Fecha de aceptación: 25/09/17

\begin{abstract}
The cleft lip and palate is a congenital craniofacial malformation that develops from embryological defects during the formation of the face. The anomalies occur mainly in the upper lip, premaxilla, palate and nasal floor. Here we introduce a case report of a patient who was admitted at 7 years old diagnosed with bilateral cleft lip and palate treated in the Craniofacial Malformations Unit of the Faculty of Dentistry of the University of Chile. The patient presented a Class III skeletal relationship, lack of development of the facial middle third, protruding position of the premaxilla, a bilateral Class III molar malocclusion with malposition and dental crowding. An orthopedic treatment of maxillary expansion was performed followed by an orthodontic treatment with fixed appliance. Following the extraction of supernumerary teeth, bone graft surgery and gingivoperiosteoplasty, she was referred to a rehabilitator who made an adhesive bridge and dental veneers, restoring aesthetics to the anterosuperior sector. Finally, removable upper and lower dental appliances were installed in the patient. Thus, satisfactory results were obtained in terms of a functional, stable and esthetic occlusion.
\end{abstract}

Keywords: Cleft palate; Mouth rehabilitation; Orthodontics. 


\section{Introducción}

El labio leporino y/o la fisura velopalatina ha sido una patología de preocupación en el área de la salud pública en Chile ${ }^{1}$ por su alta incidencia, reportándose 0,83 por cada 1.000 recién nacidos vivos (RNV) ${ }^{2}$ y en Chile 1,78 por cada 1.000 $\mathrm{RNV}^{3}$, lo que hace que cada año nazcan aproximadamente 400 niños con esta malformación. Los casos con compromiso de labio bilateral alcanzan el $25 \%$. No hay una explicación exacta de su etiología, vinculándose con factores ambientales y genéticos ${ }^{3-5}$.

Cuando se recibe en la clínica un paciente con este diagnóstico, se puede observar alteración de crecimiento y desarrollo del maxilar superior ${ }^{6}$, problemas en los dientes, con frecuencia hay ausencia o malposición de los incisivos centrales superiores, y cicatriz del labio superior. Alteraciones que comprometen fuertemente su estado psicológico y social ${ }^{7}$.

La planificación de este caso clínico fue en equipo ${ }^{8}$, teniendo presente que se terminará al fin del desarrollo y maduración del esqueleto maxilofacial, posterior al período quirúrgico ${ }^{9,10}$. Los pacientes afectados por esta patología son individuos que serán operados varias veces, ya sea si necesita una faringoplastia, injerto óseo alveolar, retoque de labio y/o rinoplastia; en algunos casos es necesaria la distracción ósea y/o cirugía ortognática ${ }^{9}$.

En el presente artículo se presenta la rehabilitación de una paciente con diagnóstico de labio leporino y fisura palatina completa tratada por el equipo de especialistas indicado.

\section{Reporte del caso}

Paciente de sexo femenino de 7 ańos de edad, consulta en el Instituto de Malformaciones Craneofaciales de la Facultad de Odontología de la Universidad de Chile con diagnóstico de fisura labiopalatina bilateral. El motivo de la consulta fue la forma alterada de la mordida y la malposición dentaria.

Al examen clínico extraoral se observó un perfil ligeramente convexo, junto a una nariz pequeña, ángulo nasolabial muy abierto, labio superior con secuela cicatrizal y cierre labial forzado (Figura 1). A nivel intraoral presenta dentición mixta primera fase. Compresión maxilar con premaxila protuída, y forma triangular de las arcadas. La cicatriz de la cirugía del paladar está presente, pero no se aprecia en toda su magnitud la separación de la premaxila de los hemimaxilares. A nivel oclusal se observa mordida bis a bis en relación a la pieza dental 1.1 e invertida en relación a la pieza 2.1. En los segmentos laterales presenta mordida abierta en relación a las piezas dentales 5.4, 5.5, 6.4, 6.5 y mordida cruzada en relación a las piezas 5.3, 6.3 y 1.6, relación oclusal molar clase III bilateral, línea media dentaria desviada levemente hacia la izquierda. Los incisivos centrales permanentes están erupcionando diastemados y girados, no se observan los incisivos laterales superiores permanentes y presenta apiñamiento dental de incisivos inferiores permanentes (Figura 2). En la radiografía panorámica se observa dentición mixta primera fase. Área apical maxi- lar disminuida con apiñamiento intraóseo. Giroversión de la pieza dental 2.1 y dientes supernumerarios bilaterales en evolución intraósea impactados, próximos a los incisivos centrales permanentes. Caninos permanentes superiores en evolución intraósea y en posición alta ( $\mathrm{Fi}$ gura 3). El examen cefalométrico reveló que la paciente presenta clase III esqueletal, falta de desarrollo del tercio medio, posición protruida de la premaxila y buen desarrollo mandibular (Figura 4).

Los objetivos terapéuticos fueron eliminar el trauma dental y alcanzar una oclusión funcional, mejorar la estética tanto facial como dental y junto a ello, su calidad de vida. Se comenzó el tratamiento ortodóncico interceptivo mediante el uso de un aparato removible con tornillo en abanico para la arcada superior con la finalidad de expandir el maxilar. Posteriormente, se confeccionó una segunda placa con tornillo central y resortes en relación a las piezas dentales 1.1 y 2.1 (Figuras 5-7). Al concluir esta etapa de tratamiento, y durante la etapa de dentición permanente, se inició el tratamiento ortodóncico con aparatología fija en ambas arcadas con el objetivo de alinear, nivelar las piezas dentarias y alcanzar una oclusión estable y funcional (Figura 8). Antes de finalizar el tratamiento ortodóncico se realizó una cirugía para extraer dientes supernumerarios adyacentes al área de la fisura bilateral, fijar la premaxila, mediante un injerto óseo y una gingivoperiosteoplastía (Figura 9).

Una vez concluido el tratamiento ortodóncico fue derivada a rehabilitación oral donde se confeccionó una prótesis parcial removible para devolver la estética en relación a los espacios desdentados correspondientes a las piezas dentales 1.2 y 2.2 (Figuras 10 y 11), y posteriormente se finalizó en base a la cementación de un puente adhesivo estético que rehabilitará de manera definitiva las piezas dentales 1.2 y 2.2. Los incisivos centrales superiores permanentes fueron incluidos en esta rehabilitación confeccionando carillas estéticas, debido a la alteración del esmalte que presentaban, tanto en forma como en color. Simultáneamente se instaló aparatos removibles de contención transversal y sagital tanto en la arcada superior como inferior (Figura 12). Mediante este tipo de rehabilitación oral combinando las técnicas antes señaladas, se logró mantener el tratamiento ortodóncico efectuado, mejorar la estabilidad oclusal y transformar la arcada dentaria de una forma triangular a una forma semicircular, mejorando de un modo significativo la estética de su sonrisa.

\section{Discusión}

La fisura labiopalatina es una de las malformaciones craneofaciales más frecuentes ${ }^{11}$. Debido a su ubicación, constituye un problema biológico y psicológico muy importante con consecuencias en el entorno familiar y social de los pacientes afectados ${ }^{1}$.

El desarrollo anormal del proceso alveolar en el área de la fisura puede generar diversas secuelas, entre las cuales destacan las alteraciones del número de dientes debido a agenesias y dientes supernumerarios, dientes ectópicos, dientes impactados, falta de desarrollo maxilar, discre- 

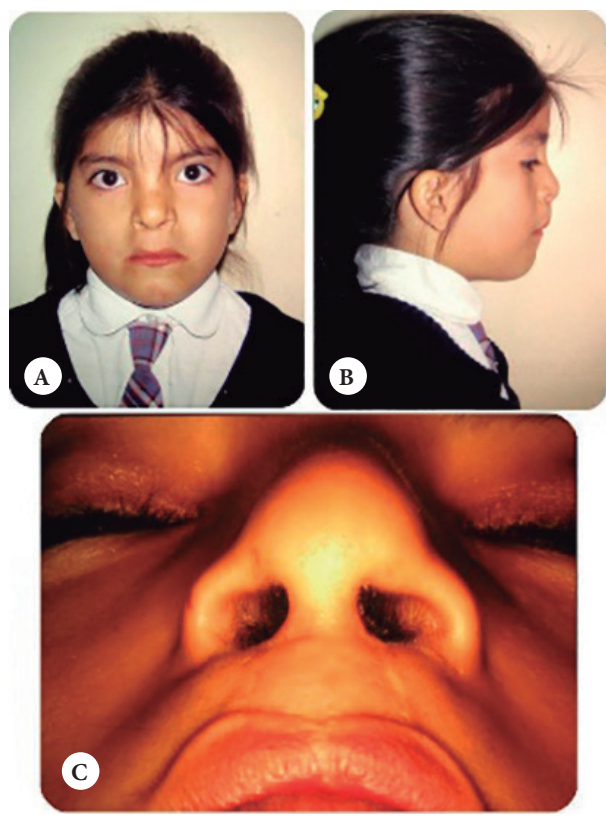

Figura 1. Fotografías extraorales iniciales: A) fotografía frontal, B) fotografía de perfil, C) Fotografía de labio superior con secuela cicatrizal.
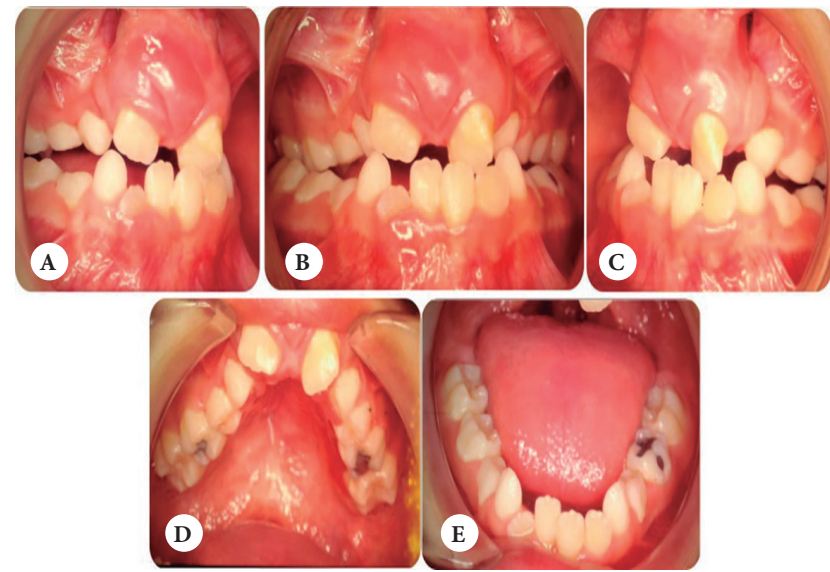

Figura 2. Fotografías intraorales iniciales de la paciente A) vista lateral derecha, B) vista frontal, C) vista lateral izquierda, D) fotografía intraoral oclusal del maxilar, E) vista oclusal mandibular.

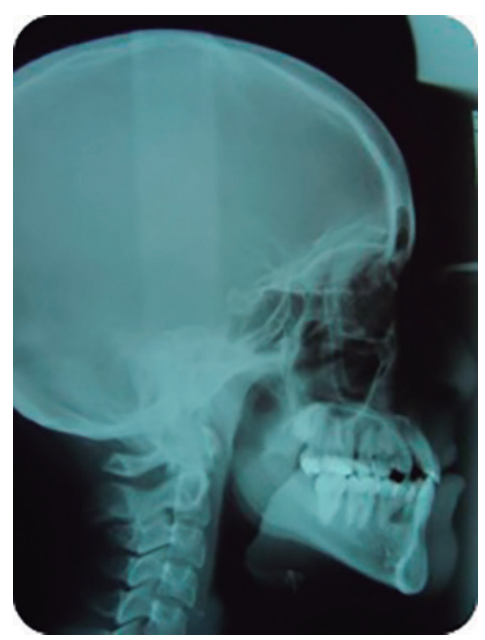

Figura 4. Telerradiografía lateral inicial.

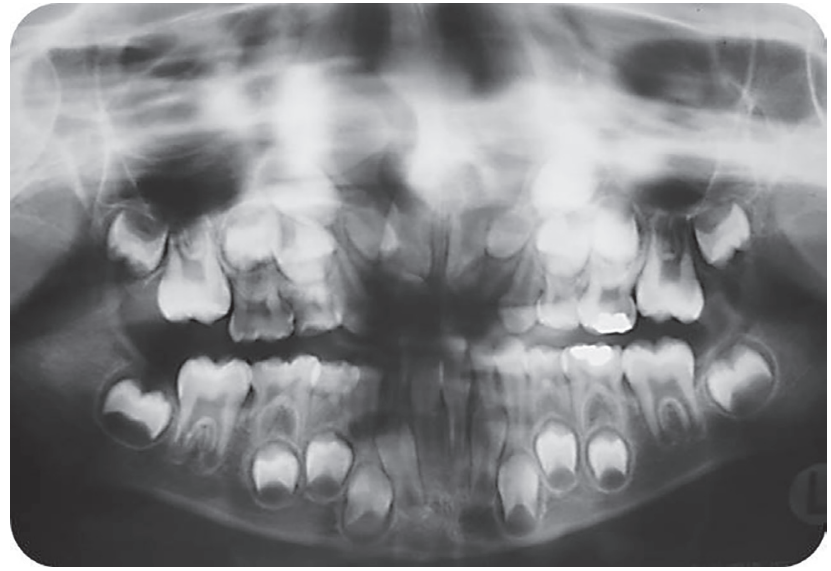

Figura 3. Radiografía panorámica inicial. Nótese presencia de dientes supernumerarios en maxilar.

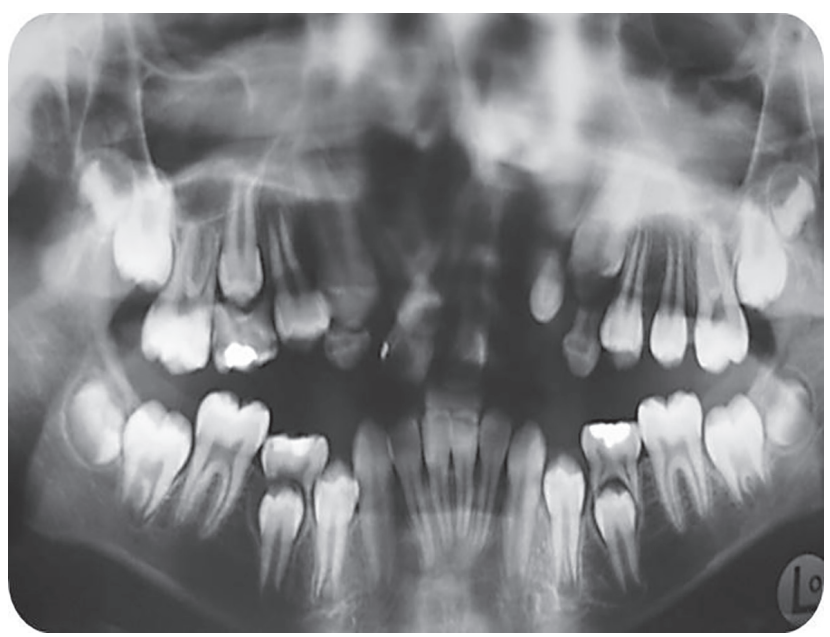

Figura 5. Radiografía panorámica previa a tratamiento ortopédico. 

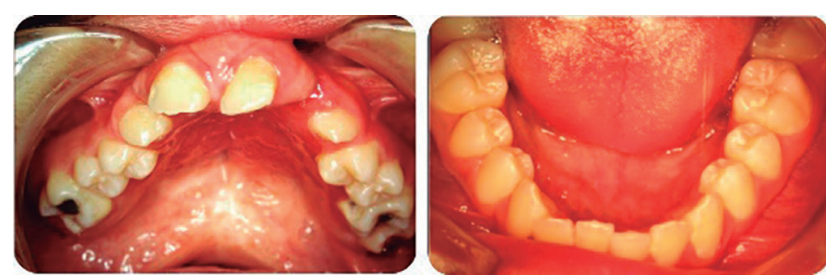

Figura 6. Fotografías intraorales previas a tratamiento ortopédico.

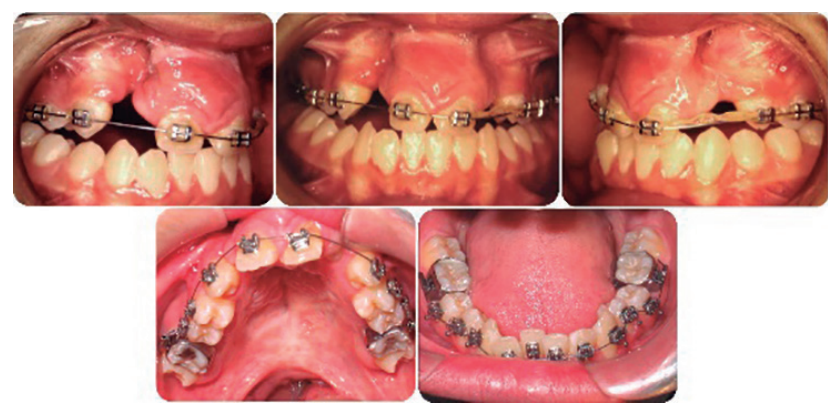

Figura 8. Fotografías intraorales durante tratamiento ortodóncico.

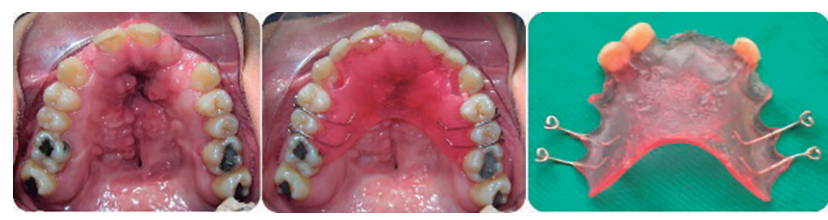

Figura 10. Rehabilitación mediante prótesis parcial removible.

pancia entre el tamaño dentario y de los maxilares, además de una falta de desarrollo del maxilar en alguno de los sentidos del espacio ${ }^{12}$. Es relevante mencionar que si bien son alteraciones que pueden estar presentes en individuos no afectados por esta patología, las anomalías de número como agenesias y dientes supernumerarios son siete veces más frecuentes en pacientes con fisura de labio y/o paladar en comparación con la población general ${ }^{11}$.

Durante la dentición temporal y mixta, tanto el cirujano como el ortodoncista deben monitorear la adecuada secuencia de erupción, exfoliación dental y los patrones de crecimiento maxilar ${ }^{13}$. La compresión maxilar y mordida cruzada, tal como se presenta en este caso clínico, son hallazgos comunes en pacientes con fisura labiopalatina ${ }^{14}$. Entre las opciones para corregir estas alteraciones se puede considerar la expansión maxilar, expansión ortodóncica asistida quirúrgicamente, distracción transpalatal y expansión quirúrgica ${ }^{15}$; y la decisión sobre el método a utilizar se debe definir considerando factores como el desarrollo de las bases óseas, la etapa de crecimiento y el grado de entrecruzamiento de las piezas dentales ${ }^{12}$. Teniendo en consideración lo antes expues-
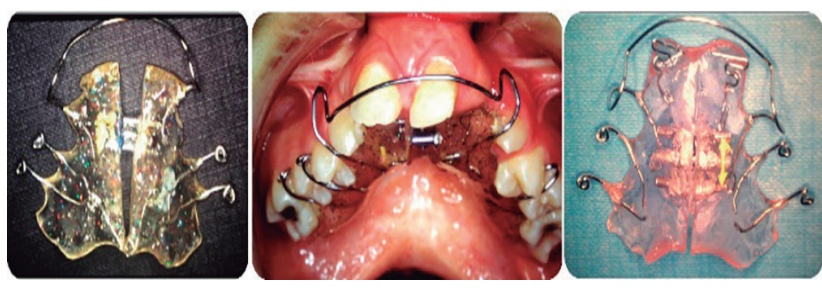

Figura 7. Aparatos ortopédicos utilizados durante el tratamiento.

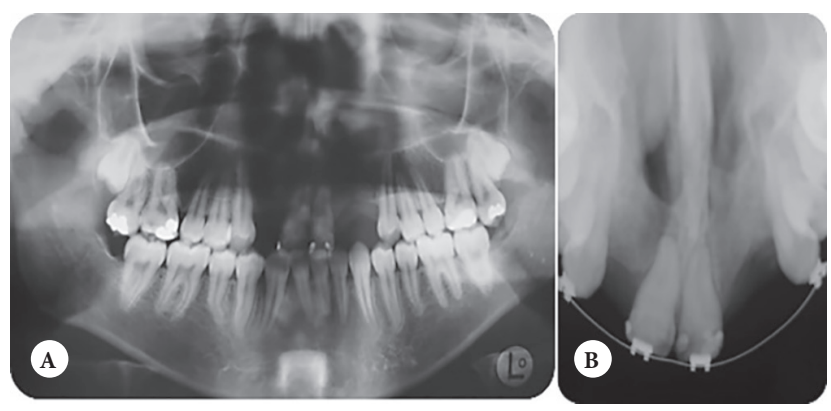

Figura 9. Radiografías posterior a injerto óseo y gingivoperiosteoplastía. A) radiografía panorámica, B) radiografía oclusal.
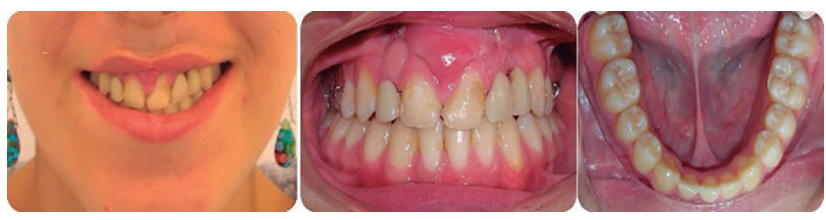

Figura 11. Fotografías extraoral e intraorales de tratamiento con prótesis parcial removible.

to, en esta paciente se determinó comenzar expandiendo el maxilar transversalmente mediante aparatos ortopédicos. Una vez que el maxilar ha alcanzado la posición óptima o la expansión con aparatos ortodóncicos ha sido suficiente, se evalúa al paciente para continuar con tratamiento ortodóncico fijo y posterior injerto alveolar ${ }^{13}$.

Diversos estudios señalan que las anomalías dentales son más frecuentes en pacientes con fisura labiopalatina que en la población en general ${ }^{14,16-18}$. Pueden ser alteraciones de número, forma, tamaño, tiempo de formación, calcificación y erupción que podrían afectar tanto la dentición temporal como la permanente. La agenesia de los incisivos laterales superiores permanentes es un problema común en estos pacientes y el manejo de esta situación implica decidir si cerrar el espacio, posicionando en su lugar el canino permanente, o crearlo para reemplazar posteriormente el diente ausente mediante una prótesis, lo que debe escogerse sobre una base individual ${ }^{12,14}$. Por otra parte, los dientes supernumerarios son la segunda anomalía más común en el área de la fisura y evaluar su presencia en esta zona es importante para determinar el periodo en que se realizará el injerto óseo alveolar secundario ${ }^{11}$. Es necesario destacar que 
pesquisar estas anomalías es fundamental en la planificación ortodóncica, quirúrgica y protésica, en función de su ubicación en el arco dental, ya que podría generar vanos desdentados que pudiesen ser cerrados o mantenidos con ortodoncia y luego rehabilitados mediante prótesis fija, removible o con implantes dentales. En el caso desarrollado, la paciente presentaba ausencia congénita de las piezas dentales adyacentes a la fisura, los incisivos laterales superiores. Pero además se pesquisó supernumerarios bilaterales contiguos al sitio de la fisura. Esta situación hizo más compleja la planificación del tratamiento, ya que se debía evaluar el tratamiento óptimo considerando ambas situaciones para alcanzar un resultado oclusal que fuera estético pero al mismo tiempo, funcional y estable. Finalmente, se decidió extraer los dientes supernumerarios y llevar a una correcta posición los caninos superiores para posteriormente rehabilitar el espacio desdentado correspondiente a los incisivos laterales permanentes ausentes.

Los pacientes con fisura labiopalatina bilateral pueden presentar protrusión de la premaxila y deformidades características que implican una gran desviación de la premaxila y los segmentos del hueso maxilar tanto derecho como izquierdo ${ }^{19}$. En el caso expuesto, esta alteración podría haber constituido un impedimento para el correcto cierre de la fisura alveolar y el tratamiento de ortodoncia, por lo cual se propuso a la paciente realizar una gingivoperiosteoplastía e injerto óseo secundario para estabilizar los segmentos maxilares y ella aceptó el plan. El objetivo del injerto es acercar los segmentos maxilares para restablecer la anatomía normal de la fibromucosa y la gingivoperiosteoplastía es la técnica más utilizada para cubrir este injerto. La adición de hueso a nivel de los dientes en la zona alveolar de la fisura mejora el soporte dental, facilita la erupción dentaria y la movilización ortodóncica de los dientes; puede utilizarse como tratamiento de las fístulas oronasales y previene su recurrencia. Otorga además, mejor soporte óseo a las alas nasales y la proyección labio-nasal, mejorando la estética facial ${ }^{15,20}$. Por último, destacar que permite restaurar la continuidad alveolar y gingival, mejorando la higiene bucal, el contorno gingival y facilitando la rehabilitación protésica posterior ${ }^{20}$.

Luego del tratamiento ortodóncico quirúrgico es común que los pacientes con fisura labiopalatina requieran una rehabilitación protésica, la cual debe planificar en conjunto tanto ortodoncista como rehabilitador. Entre las opciones podemos mencionar la rehabilitación protésica removible, fija o combinada y los implantes dentales ${ }^{14}$. La elección y ejecución debe ser planificada teniendo en consideración alcanzar una rehabilitación integral que comprenda los componentes físicos, psíquicos y emocionales del paciente. En el caso clínico desarrollado, se decidió un tratamiento rehabilitador que combinaba prótesis fija, removible y carillas altamente estéticas con el objetivo de reemplazar las piezas dentales ausentes, normalizar la oclusión y devolver la estética alterada. Dicha elección se basó en la corta edad de la paciente al momento de requerir tratamiento, presentaba escaso hueso alveolar para realizar implantes dentales y requería una contención de tratamiento de ortodoncia, por lo cual, se necesitaba un aparato de contención transversal y sagital. Además, el elevado costo de otras opciones terapéuticas y la dificultad de asistir regularmente a tratamiento debido a que vivía muy lejos de la consulta, determinaron la elección final. De este modo, la prótesis dental otorgó la estética dentaria deseada y evitó mantener zonas desdentadas. Se decidió instalar previamente una placa para evitar toda posibilidad de caries y contribuir a la independencia de la paciente para manejar su aparato removible, ante lo cual ella demostró una excelente adherencia y adaptación al tratamiento.

Una vez finalizado el tratamiento, el seguimiento de estos pacientes debe realizarse durante varios años, hasta el completo desarrollo y maduración del esqueleto. Durante todo el proceso deben ser evaluados por los diferentes profesionales quienes ejecutarán intervenciones oportunamente ${ }^{10}$. En este caso, tras el seguimiento pudimos observar que el tratamiento llevado a cabo permaneció en adecuadas condiciones funcionales y no se observaron complicaciones.

\section{Conclusiones}

Se puede concluir que el tratamiento de los pacientes con fisura labiopalatina es complejo y requiere la intervención de diversos especialistas. Estos deben interiorizarse en los diferentes aspectos a tratar e interactuar en la toma de decisiones, con la finalidad de coordinar el tratamiento de manera más efectiva y lograr una rehabilitación integral que comprenda los componentes físicos, psíquicos y emocionales del paciente.

\section{Referencias bibliográficas}

1. Vélez E, Hernández N, Pérez G, Rivera F, Soto, T. Treatment of bilateral cleft lip and palate with maxillary collapse sequelae. Case report. Rev Mex Ortodon. 2015;3(2):112-119. Doi: 10.1016/j.rmo.2016.03.034

2. Cooper ME, Ratay JS, Marazita ML. Asian oral-facial cleft birth prevalence. Cleft Palate Craniofac. J. 2006;43(5):580-9. Doi: 10.1597 / 05-167

3. Nazer J, Constanza M, Cifuentes L. Evolution of prevalence rates of orofacial clefts in a maternity of a Chilean clinical hospital. Rev Med Chile. 2010;138:567-72. Doi: S0034-9887201000050000

4. Sepúlveda G, Palomino H, Cortés J. Prevalencia de fisura labiopalatina e indicadores de riesgo: Estudio de la población atendida en el Hospital Clínico Félix Bulnes de Santiago de Chile. Rev Esp Cirug Oral y Maxilofac 2008;30(1):1725.

5. Ford A. Tratamiento actual de las fisuras labio-palatinas. Rev Med Clin Condes. 2004;15(1):3-11.

6. Madrid O, Jaramillo I, Barhoum H. Tratamiento ortodóncico de compensación de un adulto con labio y paladar hendido bilateral. Reporte de caso. Rev Estomatol. 2015;23(2):38-44.

7. Rodman RE, Tatum S. Controversies in the management of patients with cleft lip and palate. Facial Plast Surg Clin North Am. 2016;24(3):255-64. Doi: 10.1016 / j.fsc.2016.03.004 
8. Cordero E, Correa S, Pantoja R. Prevalence of patients with cleft lip and palate who were treated at the San Borja Arriarán Clinical Hospital in Santiago Chile, within the AUGE Healthcare Plan. Int J Odontostomat. 2015;9(3):469-73. Doi: 10.4067/S0718$381 X 2015000300018$

9. Ford A, Tastets M, Caceres A. Treatment of patients with cleft lip and palate. Rev Med Clin Condes. 2010;21(1):16-25. Doi: 10.1016/S0716-8640(10) 70501-0.

10. Leiva N, Nieto E, Véliz S, Valle M. Prosthetic rehabilitation in patients with cleft lip and buconasal fistula. Rev Clin Periodoncia Implantol Rehabil Oral. 2016;9(3):222-5. Doi: 10.1016/j.piro.2015.09.010.

11. Genovez GP, Cleide Carrara CF, Costa B. Tooth abnormalities of number and position in the permanent dentition of patients with complete bilateral cleft lip and palate. Cleft Palate Craniofac J. 2010;47(3):247-52. Doi: $10.1597 / 08-268.1$

12. Rioux E, Decker A, Deffrennes D. Therapeutic thoughts on the treatment of sequellae of labial-alveolar-palatal clefts in adult patients - part 2. Int Orthod. 2012;10(4):404-21. Doi: 10.1016/j.ortho.2012. 09.009

13. James JN, Costello BJ, Ruiz RL. Management of cleft lip and palate and cleft orthognathic considerations. Oral Maxillofac Surg Clin North Am. 2014;26(4):56572. Doi: 10.1016/j.coms.2014.08.007

14. Germec-Cakan D, Canter HI, Cakan U, Demir B. Interdisciplinary treatment of a patient with bilateral cleft lip and palate and congenitally missing and transposed teeth. Am J Orthod Dentofacial Orthop. 2014;145:38192. Doi: 10.1016/j.ajodo.2013.06.021.
15. Rioux E, Decker A, Deffrennes D. Therapeutic thoughts on the treatment of sequellae of labial-alveolar-palatal clefts in adult patients - part 1. Int Orthod. 2012;10: 241-60. Doi: 10.1016/j.ortho.2012.07.001

16. Gaujac C, de Souza Faco EF, Shinohara EH, de Souza Faco RA, Pereira FP, Assunção WG. Influence analysis of cleft type and supernumerary teeth eruption in the prognosis of bone graft in patients with cleft palate. J Craniofac Surg. 2014;25(5):408-11. Doi: 10.1097/ SCS.0000000000000903.

17. Ranta R. A review of tooth formation in children with cleft lip/palate. Am J Orthod Dentofacial Orthop. 1986; 90:11-8

18. Schwartz JP, Somensi DS, Yoshizaki P, Reis LLS, Lauris RCMC, Silva Filho OG, et al. Prevalence of dental anomalies of number in different subphenotypes of isolated cleft palate. Dental Press J Orthod. 2014;19(1):559. Doi: 10.1590/2176-9451.19.1.055-059.

19. Mitsukawa N, Saiga A, Morishita T, Satoh K. Special distraction osteogenesis before bone grafting for alveolar cleft defects to correct maxillary deformities in patients with bilateral cleft lips and palates: distraction osteogenesis performed separately for each bone segment. J Craniomaxillofac Surg. 2014;42(5):623-8. Doi: 10.1016/j. jcms.2013.09.003

20. Captier G, Bigorre M, Mattei L, Delestan C, Montoya P. The secondary bone grafting in cleft lip and palate: technical notes and indications about 62 cases. Ann Chir Plast Esthet. 2003;48(1):20-30. 